\title{
Giant folliculosebaceous cystic hamartoma of the upper extremity
}

Background: Folliculosebaceous cystic hamartoma (FSCH) is a rare cutaneous hamartoma consisting of dilated folliculosebaceous units invested in mesenchymal elements. These lesions have a striking predilection for the central face and scalp of adults. The vast majority of lesions present as $0.5-1.5-\mathrm{cm}$ papules or exophytic nodules. A single case of giant FSCH has been reported on the upper back.

Methods: A 32-year-old woman presented with a $(15 \mathrm{~cm}$ in greatest dimension) plaque-like, multinodular lesion on her left upper arm for several years. The lesion was clinically suspected to be a nevus sebaceus.

Results: The skin excision showed numerous dermal and subcutaneous dilated follicular structures with peripherally radiating sebaceous lobules, hair follicles, and surrounding mesenchymal elements consistent with FSCH.

Conclusion: To our knowledge, this is the second case of giant FSCH. Our case is unique for its larger size, more plaque-like growth, and location on an extremity when compared to the seminal case of giant FSCH.

Sturtz DE, Smith DJ, Calderon MS, Fullen DR. Giant folliculosebaceous cystic hamartoma of the upper extremity.

J Cutan Pathol 2004; 31: 287-290. (C) Blackwell Munksgaard 2004.
David E. Sturtz' ${ }^{1}$ David J. Smith², Marlene S. Calderon ${ }^{2}$ and Douglas R. Fullen ${ }^{1,3}$

${ }^{1}$ Department of Pathology, ${ }^{2}$ Department of Plastic Surgery, and ${ }^{3}$ Department of Dermatology, University of Michigan Hospitals, Ann Arbor, MI, USA

Douglas R. Fullen, MD, Department of Pathology, University of Michigan Hospitals, M5230, Medical Sciences I, 1301 Catherine, Ann Arbor, Ml 481090602, USA

Tel: +17347644460

Fax: +17349362756

e-mail: dfullen@umich.edu

Accepted November 19, 2003
Folliculosebaceous cystic hamartoma (FSCH) was originally described in a series of five patients by Kimura et al. in 1991. ${ }^{1}$ FSCH is a rare cutaneous hamartoma composed of dilated, cystic pilosebaceous structures surrounded by various mesenchymal elements. Lesions usually present as solitary $0.5-1.5-\mathrm{cm}$ papules or nodules, with a predilection for the central face and scalp. ${ }^{2}$ The majority of lesions are removed in adulthood, although lesions arising in infancy and childhood have been reported.

A giant FSCH was reported by Yamamoto et al. in 1994. ${ }^{3}$ The authors described a 7-cm exophytic nodule on the upper back of a 24-year-old Japanese woman. The lesion had histological features in keeping with an FSCH. As the lesion had its onset 6 months after birth, the authors considered this lesion to be a congenital variant.

We present an additional case of giant FSCH. The unique features of our case, compared to the original
FSCH, were its larger size $(15 \mathrm{~cm})$, more plaque-like growth, and occurrence on an extremity.

\section{Case report}

A 32-year-old woman was referred to a plastic surgeon in our institution for evaluation and removal of a $(15 \mathrm{~cm}$ in greatest dimension) multinodular, plaquelike growth on her left upper arm present for several years. She was otherwise in good health. The clinical impression was that this lesion was a nevus sebaceus (Fig. 1). The skin lesion was excised in toto and reconstructed.

On gross examination, the skin ellipse measured $16.7 \times 8.6 \times 1.2 \mathrm{~cm}$. It was remarkable for a $14.2 \times$ $8.0-\mathrm{cm}$ plaque-like lesion with vague multinodularity and cobblestone appearance of the skin surface. Cut surfaces revealed several nodules and cystic spaces invested in white fibrous tissue within the dermis 


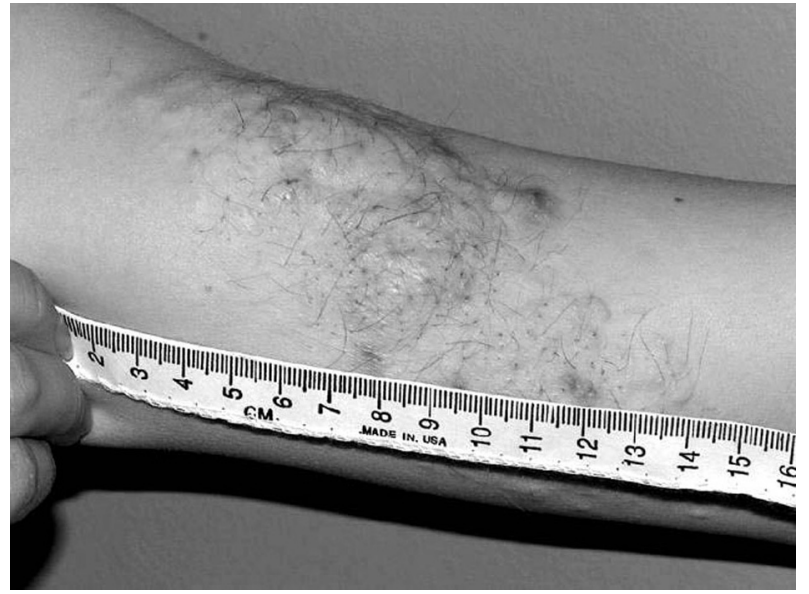

Fig. 1. Multinodular, plaque-like lesion on the upper extremity.

and subcutis (Fig. 2). Some of the cystic spaces exuded thick, paste-like pink-tan contents.

Histological examination showed several dilated, cystic follicular structures lined by stratified squamous epithelium resembling the infundibular portion of a hair follicle or the epidermis (Fig. 3). Cysts had predominantly infundibular keratinization, but occasional foci of trichilemmal keratinization were seen. The cysts were located in the dermis and subcutis. Many mature sebaceous lobules radiated outward from the cystic structures (Fig. 4). The cysts were invested in a dense fibrovascular stroma that was focally associated with slight mucin deposition and mast cells, as well as intermingled adipocytes (Fig. 5). In some areas, the stromal element appeared to be relatively well circumscribed from the adjacent normal skin. One of the cystic structures had rudimentary hair follicles and secondary cysts radiating peripherally. Some of the cysts were acutely or chronically inflamed, and bacteria were seen admixed with keratin and sebum within a few cysts.

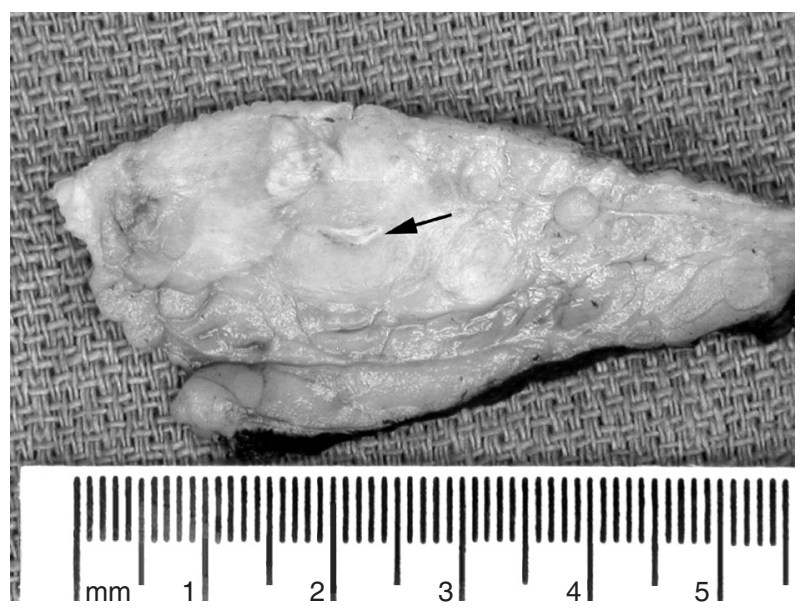

Fig. 2. Cross section showing a cystic space (arrow) with surrounding fibrosis in the dermis and subcutis.

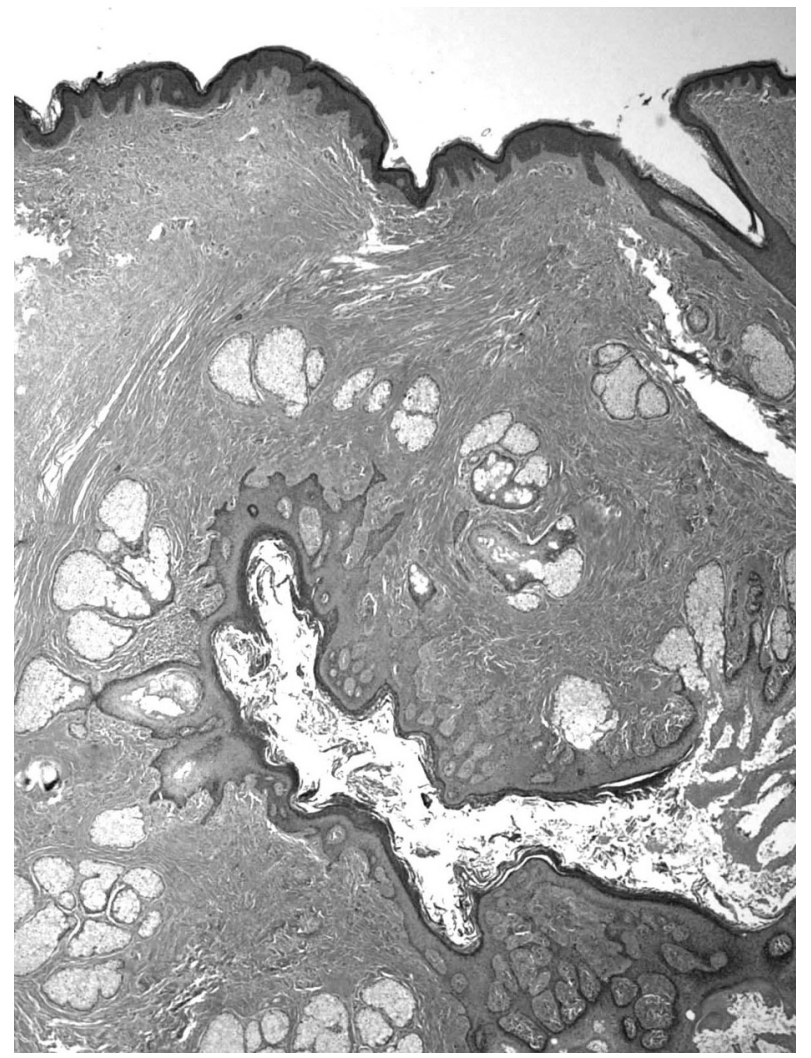

Fig. 3. Low-power view showing a dilated folliculosebaceous unit.

\section{Discussion}

There are approximately 30 cases of FSCH reported in the literature. ${ }^{1-12}$ However, Yamamoto et al. described the first and, to our knowledge, only case of giant FSCH occurring on the upper back of a Japanese woman. The large size and location were unusual for this entity which typically is $0.5-1.5 \mathrm{~cm}$ and arises primarily on the central face and scalp. The lesion they described had a polypoid, exophytic appearance. The lesion in our case was larger and,

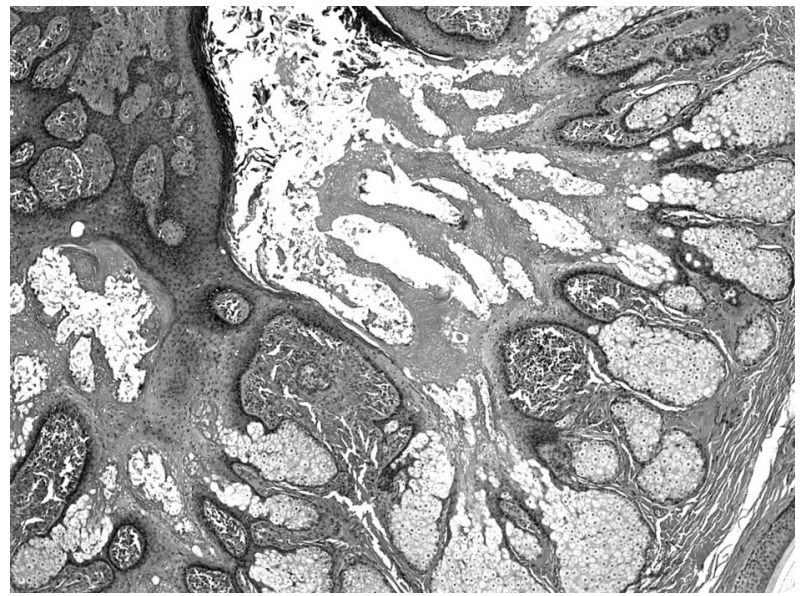

Fig. 4. Multiple sebaceous lobules radiate peripherally from a central cyst. The cyst lining demonstrates infundibular keratinization. 


\section{Giant FSCH}
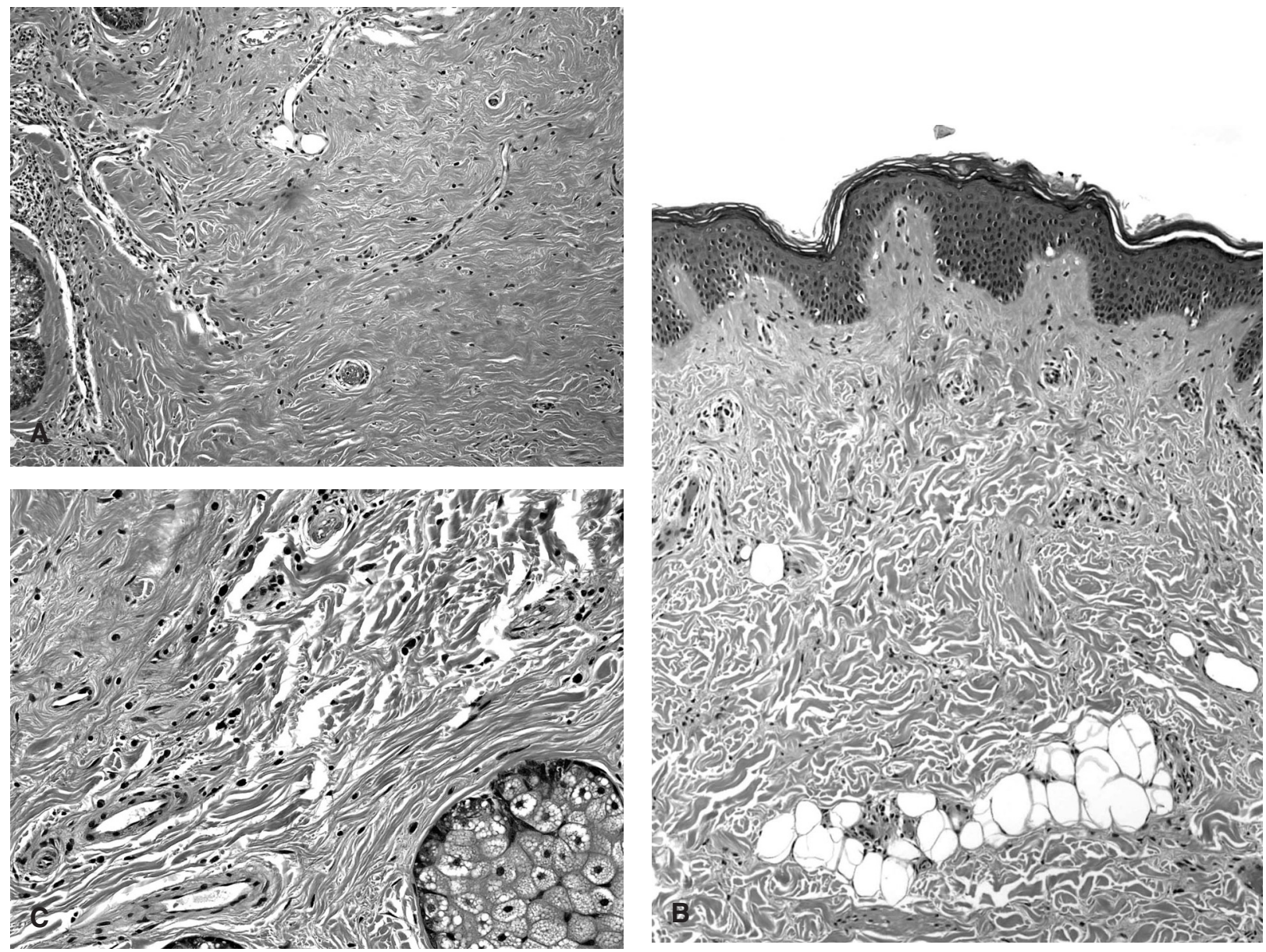

Fig. 5. Mesenchymal stromal components adjacent to pilosebaceous structures. Dense fibrous tissue with increased blood vessels (A) predominated, with occasional foci of adipocytes $(\mathrm{B})$ and mucin deposition $(\mathrm{C})$.

although multinodular, had more of a plaque-like growth pattern. Another unusual feature in our case was location of the lesion on the upper extremity. The histologic features of our case were very similar to Yamamoto's case. ${ }^{3}$

Several reports have discussed various mesenchymal elements that comprise the stromal component of FSCH. Fogt and Tahan ${ }^{4}$ reported large expanses of small, thick-walled vessels that were in either loose tufted aggregates, separated, or enveloped within the epithelial units. Donati and Balus ${ }^{5}$ presented a case with a moderate proliferation of nerve fascicles surrounding rudimentary hair follicles. Finally, Aloi et al. ${ }^{6}$ described large amounts of mucin encasing the fibroepithelial units. Our case demonstrated several mesenchymal components investing the folliculosebaceous structures. The majority of the stroma was composed of dense fibrous tissue with increased numbers of small, thick-walled blood vessels. Clusters of adipocytes were situated high in the dermis in some areas of the lesion, similar to that described by Yamamoto et al. ${ }^{3}$ Our case also demonstrated a very focal mucinous component associated with occasional mast cells. All of these features are in keeping with the stromal elements seen in FSCH. Interestingly, we did not observe a neural component in the mesenchymal stroma of this lesion, but we did not perform additional histochemical or immunohistochemical stains to further evaluate this possibility.

Recently, Schulz and Hartschuh ${ }^{7}$ have proposed that an FSCH is actually the late stage of trichofolliculoma. They noted that the differential diagnoses of FSCH include sebaceous trichofolliculoma. Two common histologic features of sebaceous trichofolliculoma are the opening of the infundibular cyst to the epidermis and the differentiation of the follicular structures toward lower follicular segments. The authors considered these features to be typical of sebaceous trichofolliculoma but not FSCH. However, these features have been documented in FSCH. The authors proposed that trichofolliculoma undergoes changes in line with the cycle of regression of a normal hair follicle and that the changes in the late stage have much in common with FSCH. These changes include infundibular cyst with sebaceous elements, inferior follicular segments in various stages of 


\section{Sturtz et al.}

regression and rest, a prominent fibrocytic and wellvascularized stroma with accumulations of adipocytes, and variable mucin deposition. The mucin deposition and adipose tissue suggested a degenerative phenomenon. Thus, Schultz and Hartschuh ${ }^{7}$ concluded that FSCH was essentially a late-stage form of sebaceous trichofolliculoma.

FSCH is a rare cutaneous hamartoma. To our knowledge, our case represents the second reported case of giant FSCH. Our case was unusual for its larger size, location on the upper extremity, and plaque-like clinical appearance compared to the original giant FSCH. Knowledge of this variant may be helpful in arriving at the correct clinical and histologic diagnosis.

\section{References}

1. Kimura T, Miyazawa H, Aoyagi T, Ackerman AB. Folliculosebaceous cystic hamartoma. A distinctive malformation of the skin. Am J Dermatopathol 1991; 13: 213.

2. Templeton SF. Folliculosebaceous cystic hamartoma: a clinical pathologic study. J Am Acad Dermatol 1996; 34: 77.
3. Yamamoto O, Suenaga Y, Bhawan J. Giant folliculosebaceous cystic hamartoma. J Cutan Pathol 1994; 21: 170.

4. Fogt F, Tahan SR. Cutaneous hamartoma of adnexa and mesenchyme. A variant of folliculosebaceous cystic hamartoma with vascular-mesenchymal overgrowth. Am J Dermatopathol 1993; 15: 73 .

5. Donati P, Balus L, Bellomo P. Folliculosebaceous cystic hamartoma. J Am Acad Dermatol 1997; 36: 502.

6. Aloi F, Tomasini G, Pippione M. Folliculosebaceous cystic hamartoma with perifollicular mucinosis. Am J Dermatopathol 1996; 18: 58.

7. Schulz T, Hartschuh W. Folliculo-sebaceous cystic hamartoma is a trichofolliculoma at its very late stage. J Cutan Pathol 1998; 25: 354 .

8. Bolognia JL, Longley BJ. Genital variant of folliculosebaceous cystic hamartoma. Dermatology 1998; 197: 258.

9. Ramdial PK, Chrystal V, Madaree A. Folliculosebaceous cystic hamartoma. Pathology 1998; 30: 212.

10. Toyoda M, Morohashi M. Folliculosebaceous cystic hamartoma with a neural component: an immunohistochemical study. J Dermatol 1997; 24: 451.

11. Yamamoto T, Ohkubo H, Nishioka K. Folliculosebaceous cystic hamartoma associated with rosacea. J Dermatol 1993; 20: 712.

12. Donati P, Balus L. Folliculosebaceous cystic hamartoma. Reported case with a neural component. Am J Dermatopathol 1993; 15: 277. 